\title{
Hepatitis $C$ virus genotype and subtype distribution in Chinese chronic hepatitis $C$ patients: nationwide spread of HCV genotypes 3 and 6
}

Wei Ju ${ }^{1 \dagger}$, Song Yang ${ }^{2 \dagger}$, Shenghu Feng ${ }^{1}$, Qi Wang ${ }^{2}$, Shunai Liu' ${ }^{1}$ Huichun Xing ${ }^{2}$, Wen Xie ${ }^{2}$, Liying Zhu ${ }^{3 *}$ and Jun Cheng ${ }^{1 *}$

\begin{abstract}
Background: Hepatitis $\mathrm{C}$ virus (HCV) genotype and subtype are related to disease progression and response to antiviral therapy. Current HCV genotype and subtype distribution data, especially for genotypes 3 and 6, are limited in China. Our purpose was to investigate the current HCV genotype and subtype distributions in chronic hepatitis $\mathrm{C}$ patients in China.

Methods: Chronic hepatitis C patients $(n=1012)$ were enrolled, and demographic information and possible transmission risk factors were collected. Serum samples were subjected to reverse-transcription polymerase chain reaction, followed by direct DNA sequencing and phylogenetic analysis of the NS5B and core/E1 regions to determine HCV genotypes/subtypes. The geographical distributions of HCV genotypes/subtypes were analyzed. Demographic information and transmission risk factors were compared between different HCV genotypes/subtypes.

Results: Four genotypes and seven subtypes of HCV were detected in 970 patients. Subtypes 1b, 2a, 3a, 6a, 3b, 6n, and 1 a were detected at frequencies of $71.96 \%, 19.90 \%, 3.20 \%, 2.16 \%, 1.96 \%, 0.41 \%$, and $0.41 \%$, respectively. Genotypes 3 and 6 showed an increasingly wide geographic distribution over time. Patients with subtypes $1 \mathrm{~b}$ and $2 a$ were older than those with $3 a, 3 b, 6 a$, and $6 n$ subtypes ( $p<0.05$ in all subtypes). More genotype 1 and 2 patients underwent blood transfusion than those with genotype 3 (all $p<0.05$ ). More genotype 3 and 6 patients had a history of intravenous drug use than those with genotypes 1 and 2 (all $p<0.05$ ).
\end{abstract}

Conclusions: Though subtypes $1 \mathrm{~b}$ and $2 \mathrm{a}$ are still the most prevalent HCV subtypes in China, genotype 3 and 6 HCV infections have already spread nationwide from southern and western China.

Keywords: Hepatitis C virus (HCV), Genotype, Phylogenetic analysis

\section{Background}

Hepatitis C virus (HCV) is a leading cause of chronic liver disease and presents a major threat to global public health. Worldwide, more than 185 million people have been infected [1], and these individuals face an increased risk of developing liver cirrhosis and hepatocellular carcinoma.

\footnotetext{
* Correspondence: zlyhmu@163.com; chengj0817@sina.cn

${ }^{\dagger}$ Equal contributors

${ }^{3}$ Department of Infectious Diseases, The Fourth Hospital of Harbin Medical University, 37 Yiyuan Street, Nangang District, Harbin 150081, China 'Institute of Infectious Diseases, Beijing Ditan Hospital, Capital Medical University, 8 East Jingshun Street, Chaoyang District, Beijing 100015, China Full list of author information is available at the end of the article
}

$\mathrm{HCV}$ can be classified into seven genotypes and at least 67 confirmed subtypes, 20 provisionally assigned subtypes, and 21 unassigned subtypes [2]. Genotypes 1, 2 , and 3 are distributed globally. In contrast, genotypes 4 , 5, and 6 are more concentrated to specific regions. Genotype 4 and subtype 5 a are mainly found in Middle Eastern countries and the northern part of South Africa, respectively. Genotype 6 is mainly found in China and Southeast Asia, especially in the Hong Kong Special Administrative Region of the People's Republic of China (HKSAR), Thailand, and Vietnam [3, 4].

\section{Ciomed Central}

(c) $2015 \mathrm{Ju}$ et al. This is an Open Access article distributed under the terms of the Creative Commons Attribution License (http://creativecommons.org/licenses/by/4.0), which permits unrestricted use, distribution, and reproduction in any medium, provided the original work is properly credited. The Creative Commons Public Domain Dedication waiver (http:// creativecommons.org/publicdomain/zero/1.0/) applies to the data made available in this article, unless otherwise stated. 
In China, the estimated prevalence of $\mathrm{HCV}$ is $0.43 \%$ [5]. A previous report indicated that subtypes $1 \mathrm{~b}$ and $2 \mathrm{a}$ were the dominant subtypes in China, although the incidences of genotype 3 and $6 \mathrm{HCV}$ infections were increasing [6]. Owing to the changing routes of transmission and increasing global travel, the geographical and genetic diversity of $\mathrm{HCV}$ is constantly changing. Recently, studies on $\mathrm{HCV}$ genotype distribution in local regions and certain patients populations were reported [7-10]. Yet nationwide $\mathrm{HCV}$ genotype and subtype distribution data of chronic hepatitis $\mathrm{C}$ patients is limited. We conducted this large-sample, multi-centered study to investigate the status quo of $\mathrm{HCV}$ genotype and subtype distribution in chronic hepatitis $\mathrm{C}$ patients in China.

\section{Results}

\section{Characteristics of the study population}

Overall, 1012 chronic hepatitis C patients were enrolled from 23 centers. Patients were located in 24 administrative units from six different geographic regions throughout China. Table 1 summarizes the demographic features and geographic origins of all enrolled patients.

\section{HCV sequence amplification, genotyping, and phylogenetic analysis}

Reliable nucleotide sequences of the NS5B and core/E1 region were obtained for 970 samples and used to construct phylogenetic trees. The phylogenetic analysis of both the NS5B and core/E1 regions revealed the presence of genotypes $1,2,3$, and 6 , while no genotype 4,5 , or 7 strains were found in this study (Table 2).

HCV genotype 1 was the most prevalent, found in 702 (72.37 \%) patients. The next most abundant genotypes were 2, 3, and 6, found in 193 (19.90\%), 50 (5.15\%), and 25

Table 1 Demographic features and geographic origins of 1012 patients enrolled in the study

\begin{tabular}{ll}
\hline Characteristic & Patients \\
\hline Gender, $\mathrm{n}(\%)$ & $506(50.0)$ \\
Male & $506(50.0)$ \\
Female & \\
Age, years & $46(12-99)$ \\
Median (range) & $48.8 \pm 14.7$ \\
Mean \pm SD & \\
Geographic origin, $\mathrm{n}(\%)$ & $111(10.97)$ \\
Northeastern China & $403(39.82)$ \\
Northern China & $40(3.95)$ \\
Central China & $275(27.17)$ \\
Eastern China & $59(5.83)$ \\
Southern China & $124(12.25)$ \\
Western China &
\end{tabular}

Table 2 Geographical distributions of HCV subtypes of 1012 patients

\begin{tabular}{llllllllll}
\hline Geographic origin & $\mathrm{n}(\%)$ & \multicolumn{1}{l}{ HCV subtypes } \\
\cline { 3 - 10 } & & $1 \mathrm{a}$ & $1 \mathrm{~b}$ & $2 \mathrm{a}$ & $3 \mathrm{a}$ & $3 \mathrm{~b}$ & $6 \mathrm{a}$ & $6 \mathrm{n}$ & $\mathrm{NA}^{\mathrm{a}}$ \\
\hline Northeastern China & $111(10.97)$ & 0 & 71 & 37 & 0 & 1 & 1 & 0 & 1 \\
Northern China & $403(39.82)$ & 1 & 270 & 90 & 13 & 7 & 3 & 0 & 19 \\
Eastern China & $275(27.17)$ & 3 & 212 & 23 & 10 & 6 & 8 & 3 & 10 \\
Central China & $40(3.95)$ & 0 & 31 & 8 & 0 & 0 & 0 & 0 & 1 \\
Southern China & $59(5.83)$ & 0 & 36 & 9 & 4 & 1 & 9 & 0 & 0 \\
Western China & $124(12.25)$ & 0 & 78 & 26 & 4 & 4 & 0 & 1 & 11 \\
Total & $1012(100)$ & 4 & 698 & 193 & 31 & 19 & 21 & 4 & 42
\end{tabular}

${ }^{\mathrm{a}} \mathrm{HCV}$ RNA could not be amplified for genotyping

(2.58 \%) patients, respectively. Evaluations of subtype revealed that the most common was $1 \mathrm{~b}$, accounting for $71.96 \%$ (698/970) of cases, followed by 2a (19.90\%; 193/ 970). Subtypes 3a, 6a, and 3b were identified in 31 (3.20\%), 21 (2.16\%), and 19 (1.96\%) patients, respectively. The least frequent subtypes were $1 \mathrm{a}$ and $6 \mathrm{n}$, with each accounting for $4(0.41 \%)$ specimens.

In phylogenetic analyses of the NS5B and core/E1 regions, the sequences of each subtype were randomly distributed with other corresponding reference sequences derived from the Los Alamos HCV database. No clear geographical clustering of Chinese isolates in this study was detected (Additional files 1, 2, 3, 4, 5, 6 and 7).

\section{Geographic distribution of HCV subtypes in China}

Subtypes $1 \mathrm{~b}$ and $2 \mathrm{a}$ were detected in all 6 geographic regions. Subtype 3b was found in all geographic regions except central China. Subtype 3a was found in 4 geographic regions, but not in central or northeastern China. Subtype 6a was found in 4 geographic regions but not in central or western China. Subtype 1a was isolated from patients in eastern and northern China. Subtype 6n strains were isolated from subjects from eastern and western China. All 7 subtypes were detected in eastern China. Other subtypes were found in northern (subtype 6), southern and western (subtype 5), and northeastern (subtype 4) China. Only 2 subtypes were detected in central China.

\section{Age versus subtype in the patient population}

Age differences were observed for some HCV subtypes. Patients infected with HCV subtype $1 \mathrm{~b}(49.8 \pm 15.0$ years $)$ and $2 \mathrm{a}(51.2 \pm 12.9$ years $)$ were older than those infected with $3 \mathrm{a}$ (36.1 \pm 9.8 years), $3 \mathrm{~b}(37.0 \pm 9.2$ years), $6 \mathrm{a}$ (38.2 \pm 12.1 years), and $6 \mathrm{n}(31.5 \pm 10.3$ years; $p<0.05$ in each for all pairwise comparisons of $1 \mathrm{~b}$ and $2 \mathrm{a}$ with $3 \mathrm{a}, 3 \mathrm{~b}, 6 \mathrm{a}$, and $6 \mathrm{n})$. There were no differences in the mean ages between subtypes $1 \mathrm{~b}$ and $2 \mathrm{a}$ (all $p>0.05$ ). There was also no difference in the mean ages between patients with subtypes $3 \mathrm{a}, 3 \mathrm{~b}, 6 \mathrm{a}$, and $6 \mathrm{n}$ ( $p>0.05$ in all cases). 
HCV transmission risk factors and different genotypes

A comparison of patients' risk factors for HCV infection and the detected HCV subtypes are shown in Table 3. A greater proportion of genotype $1(p=0.002)$ and $2(p=0.011)$ patients underwent blood transfusion compared to patients with genotype 3 infections. More genotype 1 patients underwent surgery than those with genotype $3(p=0.003)$. More genotype 2 patients underwent invasive procedures than those with genotype 1 $(p=0.015)$. Invasive procedures were performed more frequently in patients with genotype 2 and 3 infections compared to genotype 6 (all $p<0.05$ ). The history of IDU was more prevalent in genotype 3 and 6 patients than in patients with genotypes 1 or 2 (all $p<0.05$ ).

Family clustering of HCV was reported in 16 patients with subtypes $1 \mathrm{~b}(n=13)$ and $2 \mathrm{a}(n=3)$, although these patients were all from different families. The mean age was $50.1 \pm 10.9$ (range, 21-65) years and $37.50 \%(6 / 16)$ of the patients were female. They were from all geographic regions, except Southern China.

\section{Discussion}

Knowledge on HCV genotypes and subtypes is important for clinical management of chronic hepatitis $\mathrm{C}$, since discrete $\mathrm{HCV}$ genotypes respond differently to several treatment regimens $[11,12]$. Treatment with pegylated interferon (IFN) and ribavirin is still the standard of care for chronic hepatitis $C$ patients in China. However, several direct-acting antivirals (DAA) are in Phase III clinical trials. Even in the context of IFN-free DAA therapy, different $\mathrm{HCV}$ genotypes or subtypes can respond differently to the same DAA agent, leading to different resistance profiles [13]. Thus, it is imperative to clarify the current $\mathrm{HCV}$ genotype and subtype distribution in chronic hepatitis $\mathrm{C}$ patients in China.

In 2005, Lu et al. reported the HCV genotype distribution in China; they found that genotypes $1 \mathrm{~b}$ and $2 \mathrm{a}$ were the dominant subtypes and genotypes 3 and 6 were limited to southern and southwestern China [6]. Since then, many studies of genotype/subtype distribution in local regions and specific populations have been reported [7-10]. Particularly recently, Li et al. reported the epidemiology of hepatitis $C$ virus infection in highly endemic HBV areas [7]. Gu et al. conducted phylogenetic analyses of $\mathrm{HCV}$ in southern China, and revealed the constantly changing pattern of HCV genotypes in China over time [8]. Rao et al. reported the HCV genotype distribution in chronic hepatitis $\mathrm{C}$ patients in the Han ethnic population in China [10]. To provide a clear picture of the nationwide genotype distribution of $\mathrm{HCV}$ infection, we selected 23 hospitals from six geographic regions of China and enrolled 1012 naive chronic hepatitis $C$ patients. Overall, the predominance of subtype $1 \mathrm{~b}$, followed by $2 \mathrm{a}$, is in concordance with previous studies performed in different regions and different populations of China [7-10]. Previously, Lu et al. reported that genotype 6 accounted for $12.94 \%$ of $\mathrm{HCV}$ infections in China [6]. In contrast, however, the frequency of this genotype in our study was much lower $(2.58 \%)$. We consider that this difference is due to patient selection, since more patients were enrolled from southern China in the Lu et al. study, where genotype $6 \mathrm{HCV}$ infection is more prevalent than in other areas of China $[8,10,14,15]$.

Previous studies characterized genotypes 3 and 6 of $\mathrm{HCV}$ as geographically limited to western and southern China [6, 14, 15]. Recently Rong et al. reported that genotype 6a has spread from southern China and is now present nationwide [16]. The results were confirmed in this study, since we also found subtype 6a infection in eastern China, northern China, and northeastern China. However in this study, genotype $6 \mathrm{n}$ was also found in eastern China. Gentoypes $3 \mathrm{a}$ and $3 \mathrm{~b}$ were found in northeastern, northern, and eastern China. Taken together, these results reflected that genotypes 3 and 6 have already spread nationwide, possibly due to economic reasons.

The greatest diversity of HCV subtypes was found in eastern China, which may be explained by the rapid economic development and high population mobility. Only $2 \mathrm{HCV}$ subtypes were found in patients from central China, which may be related to the relatively small sample size and low population mobility in this area.

Our results support a correlation between HCV subtypes and routes of $\mathrm{HCV}$ transmission. More than $30 \%$ of individuals with HCV subtypes $1 \mathrm{~b}$ and $2 \mathrm{a}$ had a selfreported history of each blood transfusion and surgery; this was significantly higher than reports from genotype 3 patients. Among patients with a history of blood transfusion, $86.3 \%$ (297/344) received blood before 1992; in

Table 3 Reported HCV transmission risk factors associated with different HCV genotypes

\begin{tabular}{llllll}
\hline HCV genotype & Blood transfusion, $\mathrm{n}(\%)$ & Surgery, $\mathrm{n}(\%)$ & Invasive Procedure, $\mathrm{n}(\%)$ & IDU, $\mathrm{n}(\%)$ & Family history, $\mathrm{n}(\%)$ \\
\hline $1(n=702)$ & $265(37.75)$ & $262(37.32)$ & $105(14.96)$ & $22(3.13)$ & $13(1.85)$ \\
$2(n=193)$ & $67(34.72)$ & $64(33.16)$ & $43(22.28)^{\mathrm{c}}$ & $2(1.03)$ & $3(1.55)$ \\
$3(n=50)$ & $8(16.0)^{\mathrm{a}, \mathrm{b}}$ & $11(22.0)^{\mathrm{a}}$ & $11(22.0)$ & $10(20.0)^{\mathrm{a}, \mathrm{b}}$ & $0(0)$ \\
$6(n=25)$ & $4(16.0)$ & $6(24.0)$ & $1(4.0)^{\mathrm{d}, \mathrm{e}}$ & $5(20.0)^{\mathrm{d}, \mathrm{f}}$ & $0(0)$ \\
\hline
\end{tabular}

${ }^{\mathrm{a}}$ Genotype 1 vs. genotype 3: $p<0.05 ;{ }^{\mathrm{b}}$ genotype 2 vs. genotype 3: $p<0.05 ;{ }^{\mathrm{c}}$ genotype 1 vs. genotype $2: p<0.05 ;{ }^{\mathrm{d}}$ genotype 2 vs. genotype 4: $p<0.05 ;{ }^{\mathrm{e}}$ genotype 3 vs. genotype 4: $p<0.05$; ${ }^{f}$ genotype 1 vs. genotype 4: $p<0.05$; IDU, intravenous drug use 
this period, there was no screening of donated blood and blood products for $\mathrm{HCV}$. These results indicate that subtypes $1 \mathrm{~b}$ and $2 \mathrm{a}$ are more related to blood transfusion than genotype 3 , and also partially explain why subtype $1 \mathrm{~b}$ and $2 \mathrm{a}$ patients are generally older than genotype 3 patients when first diagnosed. Previous studies from other countries and regions reported that genotypes 6 and 3 are more frequent among IDU patients than in the general population [17-19]. Our current results are consistent with these reports, since more Chinese genotype 3 and 6 patients had a history of IDU when compared to genotype $1 \mathrm{~b}$ and $2 \mathrm{a}$ patients. Although genotype 3 and 6 patients are younger than genotype $1 \mathrm{~b}$ and $2 \mathrm{a}$ patients, IDU hepatitis $C$ patients have higher risk of co-infection of HIV and HBV, which may accelerate disease progression. Therefore, special attention should be paid to these IDU patients.

\section{Conclusion}

This study reveals the current HCV genotype and subtype distribution among Chinese chronic hepatitis $\mathrm{C}$ patients. At least four genotypes and seven subtypes were prevalent nationally. The distribution of HCV genotypes vary by geographic region, while subtypes $1 \mathrm{~b}$ and $2 \mathrm{a}$ remain the most prevalent in China. Genotype 3 and 6 $\mathrm{HCV}$ infections are no longer restricted to southern and western China, but are now nationwide, and this altered distribution appears closely related to IDU.

\section{Materials and methods Study population}

From June 2011 to March 2012, treatment-naive chronic hepatitis $C$ patients were randomly enrolled from 23 hospitals in China. HCV infection had been confirmed (anti$\mathrm{HCV}$ antibody and HCV RNA-positive) within 90 days prior to enrollment. Patients who had received anti-HCV therapy were excluded; no other exclusion criteria were applied. Patients were from 24 administrative units, which covered northeast China (Heilongjiang Province, Jilin Province, and Liaoning Province), northern China (Beijing Municipality, Tianjin Municipality, Hebei Province, Inner Mongolia Autonomous Region, and Shanxi Province), central China (Henan Province, Hubei Province, Hunan Province), eastern China (Anhui Province, Zhejiang Province, Shanghai Municipality, Jiangsu Province, Shandong Province, Jiangxi Province, and Fujian province), southern China (Guangdong Province), and western China (Sichuan Province, Xinjiang Uygur Autonomous Region, Qinghai Province, Shaanxi Province, and Gansu Province). Demographic information (gender, age, birthplace, etc.) and transmission risk factors (including history of blood transfusions, surgeries, invasive procedures (dental work, tattooing/piercing, etc.), or intravenous drug use (IDU), and family clustering of $\mathrm{HCV}$ infections) were collected from each patient with a face-to-face interview based on a questionnaire. Serum specimens were collected and sent to the central laboratory of the Beijing Ditan Hospital for study.

This study was approved by the ethics committee of Beijing Ditan Hospital of Capital Medical University. Written informed consent was obtained from all subjects when enrolled.

\section{HCV RNA extraction and reverse-transcription polymerase} chain reaction (RT-PCR)

HCV RNA was extracted from $140 \mu \mathrm{L}$ of each serum sample using the QIAmp Viral RNA Mini kit (Qiagen China Co., Ltd, Shanghai, China), followed by one-step RT-PCR assays with the One Step RNA PCR kit (AMV; TaKaRa Biotechnology Co. Ltd., Dalian, China). Outer sense and antisense primers derived from the NS5B and CORE/E1 region of the HCV genome were used for RT-PCR as described previously [20]. Nested PCR was performed using $10 \mu \mathrm{L}$ of the RT-PCR product using GoTaq Colorless Master Mix (Promega Biotech Co Ltd., Beijing, China), and primers as specified previously [20]. The final PCR products were confirmed by $2 \%$ agarose gel electrophoresis and visualization with ethidium bromide staining. Amplified products were sent to Beijing Centre for Physical and Chemical Analysis for sequencing with both the sense and antisense inner primers.

\section{HCV genotyping and phylogenetic analyses}

The sequences of the core/E1 and NS5B regions were aligned and edited with reference sequences of each subtype using the CLUSTALW multiple alignment program in BioEdit software [21]. HCV subtype reference sequences were derived from the Los Alamos HCV database [22]. Phylogenetic analysis was performed using the neighbor-joining method using MEGA 4, and the Kimura two-parameter method was chosen to evaluate genetic distances [23]. The reliability of the phylogenetic clustering was evaluated using bootstrap analysis with 1000 replicates. A patient's HCV subtype was determined when it was included in a defined cluster with given reference strains and with a bootstrap value above 70 .

\section{Statistical analysis}

Statistical data analyses were performed with SPSS 13.0. The continuous data were expressed as the mean \pm standard deviation (SD) or as otherwise stated, and categorical variables in absolute numbers and percentages. The student's $t$-test was used to compare the mean ages between patients infected with different HCV subtypes. The Pearson Chi-Square test was used to compare the ratio of risk factors between different genotypes. $p$ values $<0.05$ were considered statistically significant. 


\section{Ethical considerations}

This study was approved by the Ethics Committee of Beijing Ditan Hospital, Capital Medical University.

\section{Additional files}

Additional file 1: Figure S1. Subtype 1a phylogeny estimated from NS5B region sequences ( $H 77$ positions: $8244-8713$ ). Subtype 1 b sequence AB049088 and subtype 2a sequence D00944 were used as outgroups. Black circles are Chinese isolates reported in other studies and white circles label reference sequences from outside China. Sequences without a circle were from this study.

Additional file 2: Figure S2. Subtype $1 \mathrm{~b}$ phylogeny estimated from NS5B region sequences (H77 positions: 8244-8713). Subtype 1a sequence NC 004102 and subtype 2a sequence D00944 were used as outgroups. Black circles are Chinese isolates reported in other studies and white circles label reference sequences from outside China. Sequences without a circle were from this study. Black triangles represent the locations of clusters, which is not showed in detail for shortage of space.

Additional file 3: Figure S3. Subtype 2a phylogeny estimated from NS5B region sequences ( $\mathrm{H77}$ positions: 8244-8713). Subtype 1a sequence NC 004102 and subtype 2b sequence AB559564 were used as outgroups. Black circles are Chinese isolates reported in other studies and white circles label reference sequences from outside China. Sequences without a circle were from this study. Black triangle represents the location of the cluster, which is not showed in detail for shortage of space.

Additional file 4: Figure S4. Subtype 3a phylogeny estimated from NS5B region sequences ( $\mathrm{H} 77$ positions: $8244-8713)$. Subtype 1a sequence NC 004102 and subtype 3b sequence D49374 were used as outgroups. Black circles are Chinese isolates reported in other studies and white circles label reference sequences from outside China. Sequences without a circle were from this study.

Additional file 5: Figure S5. Subtype 3b phylogeny estimated from NS5B region sequences ( $H 77$ positions: 8244-8713). Subtype 1a sequence NC 004102 and subtype 3a sequence EU710463 were used as outgroups. Black circles are Chinese isolates reported in other studies and white circles label reference sequences from outside China. Sequences without a circle were from this study.

Additional file 6: Figure S6. Subtype 6a phylogeny estimated from CORE/E1 region sequences (H77 positions: 834-1318). Subtype 1a sequence NC 004102 and subtype 6b sequence D37841 were used as outgroups. Black circles are Chinese isolates reported in other studies and white circles label reference sequences from outside China. Sequences without a circle were from this study.

Additional file 7: Figure S7. Subtype $6 \mathrm{n}$ phylogeny estimated from CORE/E1 region sequences (H77 positions: 834-1318). Subtype $1 \mathrm{a}$ sequence NC 004102 and subtype 6a sequence Y12083 were used as outgroups. Black circles are Chinese isolates reported in other studies and white circles label reference sequences from outside China. Sequences without a circle were from this study.

\section{Abbreviations}

HCV: Hepatitis C virus; IDU: Intravenous drug use; RT-PCR:

Reverse-transcription polymerase chain reaction; DAA: Direct-acting antivirals.

\section{Competing interests}

All authors declare that they have no competing interests.

\section{Authors' contributions}

CJ and ZL contributed to the study design, critical review of the manuscript, and approval of the final draft. JW and YS contributed to data collection, lab testing, and drafting of the manuscript. FS, WQ, and LS contributed to data collection and lab testing. $\mathrm{XH}$ and $\mathrm{XW}$ contributed to critical review of the manuscript. All authors read and approved the final manuscript.

\section{Acknowledgements}

We are grateful to all the clinicians from the following 23 hospitals for their assistance in collecting serum samples and questionnaires from patients enrolled in this study: Beijing Ditan Hospital Capital Medical University; First Affiliated Hospital of the Medical College, Shihezi University; First Teaching Hospital of Tianjin University of TCM; Hospital for Infectious Diseases, Mudanjiang; Hospital for Infectious Diseases, Wuxi; Jiangsu Province Hospital; People's Hospital of Guangshan County, Henan Province; People's Hospital of Taihe County, Anhui Province; People's Hospital of Jincheng City, Shanxi; Ruijin Hospital, Shanghai Jiaotong University School of Medicine; Shenzhen Third People's Hospital; The Affiliated Hospital (group) of Putian University; The First Affiliated Hospital of Baotou Medical College; The First Affiliated Hospital of Wenzhou Medical College; The First Hospital of Lanzhou University; The First Hospital of Shanxi Medical University; The Fourth Hospital of Harbin Medical University; The Second Hospital of Shandong University; The Third Hospital of Hebei Medical University; The Third Hospital of Qinhuangdao; Tianjin Third Central Hospital; Xinjiang Uyger Municipal People's Hospital; Zhenjiang Third People's Hospital. The study was supported by Beijing Municipal Administration of Hospitals Clinical Medicine Development of Special Funding Support (No.ZY201402). We would like to thank Editage for English language editing.

\section{Author details}

${ }^{1}$ Institute of Infectious Diseases, Beijing Ditan Hospital, Capital Medical University, 8 East Jingshun Street, Chaoyang District, Beijing 100015, China. ${ }^{2}$ Center of Hepatology, Beijing Ditan Hospital, Capital Medical University, 8 East Jingshun Street, Chaoyang District, Beijing 100015, China. ${ }^{3}$ Department of Infectious Diseases, The Fourth Hospital of Harbin Medical University, 37 Yiyuan Street, Nangang District, Harbin 150081, China.

Received: 9 June 2015 Accepted: 10 July 2015

Published online: 25 July 2015

\section{References}

1. Mohd HK, Groeger J, Flaxman AD, Wiersma ST. Global epidemiology of hepatitis C virus infection: new estimates of age-specific antibody to HCV seroprevalence. Hepatology. 2013;57:1333-42.

2. Smith DB, Bukh J, Kuiken C, Muerhoff AS, Rice CM, Stapleton JT, et al Expanded classification of hepatitis $C$ virus into 7 genotypes and 67 subtypes: updated criteria and genotype assignment web resource. Hepatology. 2014;59:318-27.

3. Gower E, Estes C, Blach S, Razavi-Shearer K, Razavi H. Global epidemiology and genotype distribution of the hepatitis C virus infection. J Hepatol. 2014;61:S45-57.

4. Sievert W, Altraif I, Razavi HA, Abdo A, Ahmed EA, Alomair A, et al. A systematic review of hepatitis $C$ virus epidemiology in Asia, Australia and Egypt. Liver Int. 2011;31:61-80.

5. Cui Y, Jia J. Update on epidemiology of hepatitis B and C in China. J Gastroenterol Hepatol. 2013;28:7-10.

6. Lu L, Nakano T, He Y, Fu Y, Hagedorn CH, Robertson BH. Hepatitis C virus genotype distribution in China: predominance of closely related subtype $1 \mathrm{~b}$ isolates and existence of new genotype 6 variants. J Med Virol. 2005;75:538-49.

7. Li D, Long Y, Wang T, Xiao D, Zhang J, Guo Z, et al. Epidemiology of hepatitis $C$ virus infection in highly endemic HBV areas in China. PLoS One. 2013;8:e54815.

8. Gu L, Tong W, Yuan M, Lu T, Li C, Lu L. An increased diversity of HCV isolates were characterized among 393 patients with liver disease in China representing six genotypes, 12 subtypes, and two novel genotype 6 variants. J Clin Virol. 2013;57:311-7.

9. Pan S, Rao Y, Li J, Yang H, Tang J, Zhong R, et al. Hepatitis C virus genotype diversity in Shanghai, China. Arch Virol. 2013;158:187-91.

10. Rao H, Wei L, Lopez-Talavera JC, Shang J, Chen H, Li J, et al. Distribution and clinical correlates of viral and host genotypes in Chinese patients with chronic hepatitis C virus infection. J Gastroenterol Hepatol. 2014;29:545-53.

11. European Association for the Study of the Liver. EASL recommendations on treatment of hepatitis C 2014. J Hepatol. 2014;61:373-95.

12. Omata M, Kanda T, Yu M-L, Yokosuka O, Lim S-G, Jafri W, et al. APASL consensus statements and management algorithms for hepatitis $C$ virus infection. Hepatol Int. 2012;6:409-35. 
13. Poveda E, Wyles DL, Mena A, Pedreira JD, Castro-Iglesias A, Cachay E. Update on hepatitis $C$ virus resistance to direct-acting antiviral agents. Antiviral Res. 2014;108:181-91.

14. Zhou Y, Wang X, Mao Q, Fan Y, Zhu Y, Zhang X, et al. Changes in modes of hepatitis $C$ infection acquisition and genotypes in southwest China. J Clin Virol. 2009:46:230-3.

15. Tan Y, Wei QH, Chen L, Chan PC, Lai WS, He ML, et al. Molecular epidemiology of HCV monoinfection and HIV/HCV coinfection in injection drug users in Liuzhou, Southern China. PLoS One. 2008;3:e3608.

16. Rong X, Xu R, Xiong H, Wang M, Huang K, Chen Q, et al. Increased prevalence of hepatitis $\mathrm{C}$ virus subtype $6 \mathrm{a}$ in China: a comparison between 2004-2007 and 2008-2011. Arch Virol. 2014;159:3231-7.

17. Zhou DX, Tang JW, Chu IM, Cheung JL, Tang NL, Tam JS, et al. Hepatitis C virus genotype distribution among intravenous drug user and the general population in Hong Kong. J Med Virol. 2006;78:574-81.

18. Aceijas C, Rhodes T. Global estimates of prevalence of HCV infection among injecting drug users. Int J Drug Policy. 2007;18:352-8.

19. Garten RJ, Lai S, Zhang J, Liu W, Chen J, Vlahov D, et al. Rapid transmission of hepatitis $\mathrm{C}$ virus among young injecting heroin users in Southern China. Int J Epidemiol. 2004;33:182-8.

20. Demetriou VL, van de Vijver DA, Kostrikis LG. Molecular epidemiology of hepatitis C infection in Cyprus: evidence of polyphyletic infection. J Med Virol. 2009;81:238-48.

21. Thompson JD, Higgins DG, Gibson TJ. CLUSTAL W: improving the sensitivity of progressive multiple sequence alignment through sequence weighting, position-specific gap penalties and weight matrix choice. Nucleic Acids Res. 1994;22:4673-80.

22. Kuiken C, Yusim K, Boykin L, Richardson R. The Los Alamos hepatitis C sequence database. Bioinformatics. 2005;21:379-84.

23. Kumar S, Nei M, Dudley J, Tamura K. MEGA: a biologist-centric software for evolutionary analysis of DNA and protein sequences. Brief Bioinform. 2008:9:299-306.

\section{Submit your next manuscript to BioMed Central and take full advantage of:}

- Convenient online submission

- Thorough peer review

- No space constraints or color figure charges

- Immediate publication on acceptance

- Inclusion in PubMed, CAS, Scopus and Google Scholar

- Research which is freely available for redistribution 